\title{
МЕТОДЫ НЕЙРОЭВОЛЮЦИИ СЕТЕЙ ПРЯМОГО РАСПРОСТРАНЕНИЯ
}

\section{ю. И. Давидюк}

${ }^{1}$ Магистр технических наук, старший преподаватель кафредры интеллектуальных инфоормационных технологий учреждения образования «Брестский государственный технический универсuтет», Брест, Беларусь, e-mail: yuidavidyuk@g.bstu.by

\begin{abstract}
Ресрерат
Рассматриваются различные методы оптимизации архитектуры и параметров нейронных сетей прямого распространения эволюционными алгоритмами.

Проведен анализ методов нейроэволюции, описаны основные свойства и применимость разнообразных вариантов эволюционной оптимизации с прямым кодированием хромосом для различных архитектур нейронных сетей прямого распространения.

Для каждого метода нейроэволюции приведены используемые операторы генетического алгоритма, описаны достоинства, недостатки и особенности их применения. Результаты сравнения представлены в табличном виде.
\end{abstract}

Ключевые слова: генетический алгоритм, нейронная сеть, эволюционное программирование, нейроэволюция.

\section{NEUROEVOLUTION METHODS FOR FEEDFORWARD NEURAL NETWORKS}

\section{Abstract}

\section{Y. I. Davidyuk}

Various optimization methods and parameters of feedforward neural networks by evolutionary algorithms are considered.

The analysis of neuroevolutionary methods, basic properties and applicability of various variants of evolutionary optimization with direct coding of chromosomes for various architectures of feedforward neural networks are presented.

Operators of the genetic algorithm, advantages, disadvantages and features of their application are described for each method of neuroevolution. The comparison results are presented in tabular form.

Keywords: neural network, genetic algorithm, evolutionary programming, neuroevolution.

\section{Введение}

Все больше повседневные задачи различных отраслей автоматизируются, такие как распознавание объектов, идентификация, прогнозирование, фильтрация, восстановление, классификация и т. д. Все эти задачи связаны с аппроксимацией нелинейных функций. Использовать традиционные методы аппроксимации, например метод наименьших квадратов, из-за отсутствия полной информации о виде нелинейности функции недостаточно эфффективно. В таких случаях рассматривается использование нейросетевых технологий, искусственных нейронных сетей (ИНС). ИНС являются универсальными аппроксиматорами, что дает возможность восстановить очень сложные непрерывные нелинейные функции с заданным отклонением.

Наибольшее распространение при решении такой задачи получили ИНС прямого распространения без адаптивного изменения топологии, но существуют некоторые сложности при их использовании, а именно: выбор топологии сети, выбор параметров сети, настройка весовых коэффициентов. Задача выбора и определения топологии ИНС является комбинаторной, а настройка параметров ИНС, то есть поиск оптимальных параметров, осуществляется в непрерывном пространстве с помощью классических методов оптимизации. Для обучения ИНС применяются, как правило, методы, требующие вычисления градиента, но они в свою очередь имеют недостатки.

Попытки устранить недостатки традиционных методов привели к изучению возможности применения эволюции, а точенее эволюционных вычислений в данной области, так появилась отдельная область - нейроэволюция, а сети, использующие нейроэволюцию, получили название эволюционирующих ИНС (ЭИНС).

Использование в ЭИНС эволюции и обучения, позволяющих изменять структуру сети, ее параметры и алгоритмы обучения без внешнего вмешательства, делает данные сети наиболее приспособленными для работы в нестационарных условиях и наличии неопределенности относительно свойств исследуемого объекта и условий его функционирования. Основным преимуществом использования эволюционных алгоритмов (ЭА) в качестве алгоритмов обучения является то, что многие параметры ИНС могут быть закодированы в геноме и определяться параллельно. Более того, в отличие от большинства алгоритмов оптимизации, предназначенных для пошагового решения задачи, ЭА оперируют с множеством решений популяцией, что позволяет достичь глобального экстремума, не застревая в локальных. При этом информация о каждой особи популяции кодируется в хромосоме (генотипе), а получение решения (фенотипа) осуществляется после эволюции (отбора, скрещивания, мутации) путем декодирования.

Применение традиционных методов исследования для этих видов задач достаточно часто не дает желаемого результата, так как вычислительные затраты растут вместе с размерностью задачи в экспоненциальной зависимости. В связи с этим для решения практических задач применяются эвристические методы, являющиеся более гибкими и эфффективными и требующие гораздо меньших вычислительных затрат, даже не достигая глобального оптимального решения. К таким методам оптимизации относят метод имитации отжига, ИНС, ЭА, алгоритм пчелиного роя, муравьиные алгоритмы и т. д.

Анализ существующих интеллектуальных систем обработки информации показывает, что совместное использование нейронных сетей и эволюционных алгоритмов может повысить скорость и качество настройки параметров ИНС, для быстрого получения достаточно хороших результатов за короткий промежуток времени при необходимости. Использовать нейроэволюционные методы можно по-разному: настройка только весов нейронной сети, настройка только топологии сети, настройка остальных параметров сети, а также возможная их комбинация [1].

На данный момент все больше набирают популярность методы обучения ИНС с подкреплением [2], но пока не найдены удовлетворительные решения для определенного круга задач: прогнозирование курса валют, состояние биржевых рынков и т. д. Исходя из этого можно сделать предположение, что именно нейроэволюционные методы настройки ИНС, которым не нужна обучающая выборка с эталонами, могут способствовать улучшению результатов за счет рассмотрения множества решений и оценки их поведения за более короткий промежуток времени.

При использовании ЭА в ИНС основными проблемами являются выбор метода кодирования, генетических операторов. Кодирование может быть прямым, когда хромосома содержит всю информацию о топологии сети (слои, нейроны, связи) и косвенным , когда в хромосоме кодируется только число слоев и число нейронов в каждом слое, что существенно уменьшает длину хромосомы. В зависимости от выбранного способа кодирования существуют различные методы нейроэволюции.

Следует провести подробный анализ существующих методов нейроэволюции, определить возможности каждого, выявить достоинства и недостатки для дальнейшей разработки модифицированного метода нейроэволюции с использованием не только универсального генетического алгоритма, но и существующих определенных 
Вестник Брестского государственного технического университета. 2021

алгоритмов: рой частиц, стая серого волка, гравитационный алгоритм, стая летучих мышей и т. д.

Эволюционная оптимизация с прямым кодированием хромосом

Эволюционная оптимизация топологии сети состоит из двух фаз: кодирование, реализация ЭА. Обычно настройка параметров осуществляется после того, как получена приемлемая топология сети, что характеризуется допустимым значением фитнесс-функции (функции приспособленности) [3]. Эволюционная настройка весовых параметров сети также требует задания способа их кодирования (бинарное или вещественное), влияющего на выбор операторов скрещивания (кроссинговера) и мутации.

Инициализации весов производится случайным образом, в связи с чем при применении конкретного алгоритма обучения может возникать ошибочная оценка приспособленности:

- При одних и тех же начальных условиях различные алгоритмы обучения могут приводить к различным результатам.

- Один и тот же генотип после обучения может иметь разную приспособленность. Попытка получения усредненных результатов путем обучения с разными начальными условиями приводит к резкому возрастанию вычислительных затрат.

Для получения более точных результатов стоит рассматривать одновременную эволюцию архитектуры и параметров ИНС (весов, порогов). Нейроны осуществляют нелинейные преобразования сигналов в соответствии с видом их функций активаций, поэтому выбор этих фуннций играет не последнюю роль в сложности вычислений. Чаще всего для упрощения предполагается, что функции нейронов одинаковы и выбраны экспертом.

Информация о функцции активации может быть закодирована в хромосоме, а может и отсутствовать вовсе. В настоящее время существует большое число методов настройки параметров сети, отличающихся объемом используемой информации, влияющим как на динамические свойства алгоритмов, так и на их вычислительную сложность. Рассмотрим существующие методы нейроэволюции.

CNE (Conventional NeuroEvolution, стандартная нейроэволюция) метод, модифицирующий весовые коэффициенты нейросети и не затрагивающий ее структуру. Метод CNE - это одна из первых успешных реализаций нейроэволюции, предложенная Ричардом Белю, Джоном Маккинри и Николасом Шраудольфом в 1990 г. [4]. Несмотря на простоту, CNE внес значительный вклад в развитие методов нейроэволюционной оптимизации. Структура ИНС, количество нейронов и все параметры за исключением весовых коэффициентов задаются в методе заранее.

Для оптимизации как правило выбираются полносвязные ИНС. К достоинствам метода относятся: простота реализации преобразований генотип-фенотип, высокая скорость работы за счет компактного представления генотипов. У метода CNE также есть и недостатки. Так, существует высокая вероятность сходимости метода к локальному оптимуму. Метод требует предварительного задания топологии нейросети, количества нейронов и связей, а также обладает низкой масштабируемостью и не предназначен для решения сложных задач.

EPNet (Evolutionary Programming Network, эволюционное программирование нейросети) - метод, единовременно оптимизирующий топологию и весовые коэффициенты ИНС, впервые был предложен Яо и Лю в 1997 г. [5].

Метод оптимизирует только нейросети прямого распространения с сигмоидными функциями активации. Метод оперирует пятью видами мутации для настройки топологии ИНС: удаление скрытых нейронов, удаление связей, добавление нейронов и связей, настройка весовых коэффициентов. Данный метод использует прямую схему кодирования хромосом с двумя матрицами (матрица связей, матрица весовых коэффициентов).

Достоинством метода является компромисс между структурной сложностью формируемых решений и временными затратами работы. Метод предполагает, что оператор мутации топологии ИНС задействуется только в случае, когда ошибка вывода перестает уменьшаться (нейросеть попадает в точку локального оптимума). В первую очередь осуществляется удаление нейронов или связей, а в случае неудачи - добавления данных структурных элементов.

Так как в методе EPNet в качестве способа обучения ИНС используется метод обратного распространения ошибки (МОРО), то на него распространяются и проблемы, свойственные градиентным методам (схождение в локальном минимуме, настройка размера шага обучения). Также для данного способа обучения с учителем

необходимо подготовить обучающую выборку, а использование матриц для реализации прямой схемы кодирования и нескольких вспомогательных векторов делает метод ресурсоемким.

SANE (Symbiotic Adaptive Neuro-Evolution, адаптивная симбиотическая нейроэволюция) - метод настройки топологии ИНС прямого распространения с одним скрытым слоем, основанный на кооперативной коэволюции, был разработан Дэвидом Мориарти и Ристо Микклайненом в 1997 г. [6]. Используется в сетях, решающих задачи управления в динамической среде.

Особи популяции нейронов закодированы в виде бинарных хромосом, содержащих связи нейронов. В методе реализован генетический алгоритм с использованием операторов мутации и кроссинговера. Кооперативная модель коэволюция позволяет разбивать сложную задачу на более мелкие части (каждая особь популяции представляет собой только определенную часть) и оптимизировать их, что упрощает решение задач, обладающих высокой вычислительной и структурной сложностью. Полное решение формируется из множества особей, содержащих оптимизированное решение подзадачи.

Метод SANE параллельно оперирует двумя популяциями: популяцией нейронов, содержащих информацию о связях с другими нейронами, и популяцией шаблонов нейросетей. Эволюция в популяции нейронов предназначена для поиска жизнеспособных элементов ИНС, в то время как эволюция шаблонов нейросетей направлена на формирование эффрективных комбинаций из имеющихся элементов.

Достоинством метода SANE является использование специализированных нейронов и их постоянное нахождение в популяции, что позволяет успешно бороться с преждевременной сходимостью и нахождением локального оптимума. К недостаткам метода можно отнести ограничение топологии ИНС, отсутствие оптимизации пороговых значений и вида функции активации.

На основе данного метода был разработан метод ESP (Enforced SubPopulations, усиленные субпопуляции) с введением рекуррентных связей между нейронами.

NEAT (Neuro-Evolution by Augmenting Topologies, нейроэволюционный метод расширения топологий) - метод, оптимизирующий веса и последовательно усложняющий структуру ИНС. Метод был разработан Кеном Стэнли в 2002 г. [7].

В методе используется прямая схема кодирования. Исходная популяция генерируется из полносвязных нейросетей, состоящих из входного и выходного слоев, количество нейронов в которых определяется заранее. За основу структуры генотипа в методе взят список связей. Как показано на рис. 1, каждая связь хранит индексы двух нейронов (источник и приемник сигнала), вес связи, бит активности, указывающий активна ли данная связь, и инновационное число для определения аналогичных генов при кроссинговере.

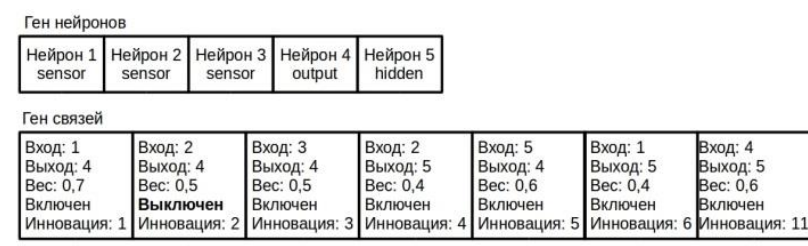

Рисунок 1 - Генотип нейронной сети метода NEAT

Метод NEAT реализует два оператора мутации для раздельной модификации весов и структуры; для каждого весового коэффициента вероятность мутации фиксирована. Структурные мутации увеличивают размер генотипа за счет добавления новых генов. Новый ген добавляет либо связь для двух ранее не соединенных нейронов, либо новый нейрон: при этом существующая связь становится не активной и разбивается на две - вход и выход нового нейрона (см. рис. 2).

Оператор кроссинговера выполняется на гомологичных генах, то есть необходимо выполнить выравнивание генов перед кроссинговером (см. рис. 3).

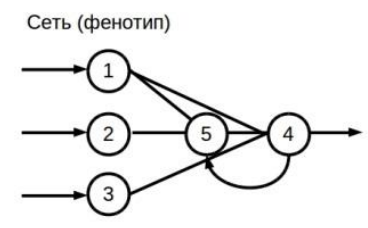

Рисунок 2 - Добавление нового нейрона 5 


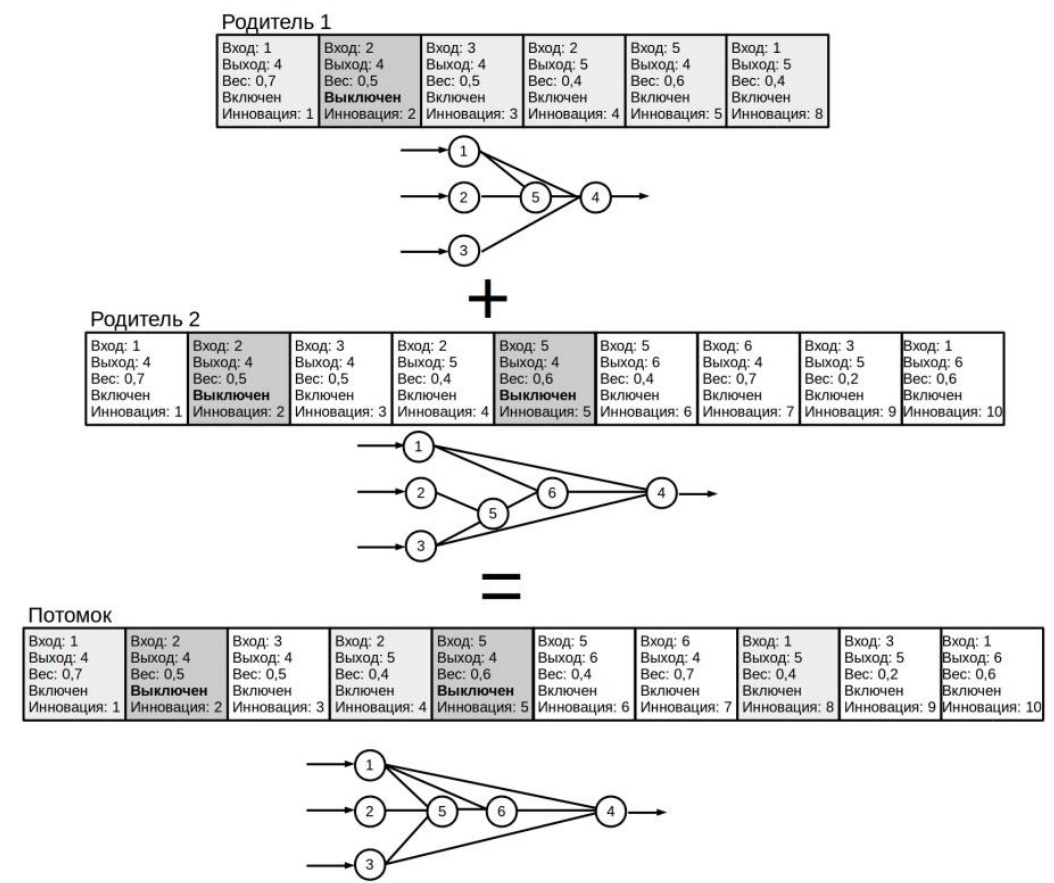

Рисунок 3 - Кроссинговер метода NEAT

Одной из особенностей метода NЕAT является использование инновационных чисел для отслеживания хронологии изменений. Гены с одинаковыми инновационными числами выравниваются и формируют генотип для следующего поколения двумя возможными способами: смешиванием случайным образом подходящих генов либо усреднением весов связей. На этапе кроссинговера задается вероятность повторной активации для неактивированных генов.

Метод NEAT работает с видами внутри популяции и раздельным вычислением приспособленности каждого вида, что позволяет сохранять генетическое разнообразие.

К достоинствам метода NEAT относятся защита инноваций историческими маркерами и поддержание разнообразия популяции, что позволяет решить проблемы преждевременной сходимости и незащищенности инноваций. К недостаткам можно отнести ограничение пространства поиска и высокая ресурсоемкость.

NES (Novel Evolutions Strategy - новая эволюционная стратегия) метод, при котором топология ИНС фиксированная, изменению подвержены только параметры данной ИНС, был предложен Каер Ассадом в 2003 г. [8]. Метод использует два генетических оператора: арифметический оператор кроссинговера, основанный на субпопуляциях (см. рис. 4) и оператор мутации, изменяющий свои параметры по функции Гаусса в зависимости от продолжительности эволюции (Time-Variant Mutation, TVM).

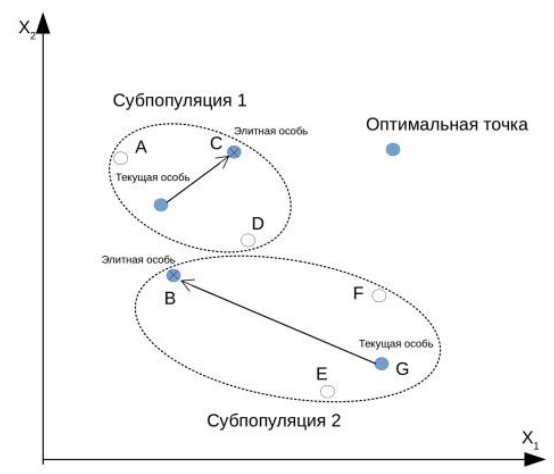

Рисунок 4 - Пример арифметического оператора кроссинговера Subpopulation-Based Max-mean Arithmetical Crossover

Достоинством метода является динамичность: многие параметры генетических операторов корректируются в ходе эволюции. Недостатком метода является невозможность изменения топологии ИНС.
EANT (Evolutionary Acquisition of Neural Topologies, эволюционное модифицирование топологий нейронных сетей) - метод, использующий гибридную схему кодирования CGE (common genetic encoding, обобщенная схема кодирования генов). Метод был разработан Йохансом Кассауном и Джеральдом Соммером в 2005 г. [9].

Схема CGE используется при раздельном изменении параметров сети и ее топологии. Схема обладает двумя важными свойствами: полнотой и замкнутостью. Благодаря использованию CGE в методе EANT удалось исключить такой этап, как декодирование хромосом. Схема CGE определяет генотип ИНС как линейную последовательность генов, каждый из которых может представлять одну структурную единицу: вход, узел или джампер. Вход - ген, обозначающий входной нейрон. Узел - ген, обозначающий нейрон, у которого имеется четыре параметра: значение весового коэффициента, текущее значение функции активации, глобальный уникальный идентификатор и количество входных связей. Джампер - ген связи, хранящий ссылки на два узла (входной и выходной), и глобальный уникальный идентификатор нейрона, с которым соединен джампер. Джампер добавляется в генотип в результате применения оператора структурной мутации.

К достоинствам метода EANT относятся компактность представления генотипа, отсутствие этапа декодирования и, как следствие, высокая скорость работы. В методе EANT отсутствует оператор кроссинговера и структурной мутации для удаления нейронов, что ограничивает пространство генетического поиска, что, в свою очередь, является его недостатком. Следует отметить, что данный метод целесообразно использовать в системах, имеющих ограничение по времени на решение задач.

ENS $^{3}$ (Evolution of Neural Systems by Stochastic Synthesis, эволюция нейронных систем путем стохастического синтеза) - метод, основанный на совместной эволюции биологических видов, взаимодействующих в экосистеме, т. е. приспособленность отдельных особей зависит от других особей популяции. Впервые был предложен и разработан Фрэнком Паземаном и Ульфом Дикманом в 1997 г. [10].

Метод ENS 3 применим для ИНС, которые используют сигмоидную функцию активации, метод не ограничивает количество нейронов в каждом слое и количество слоев, а также данный метод применим для любой топологии ИНС. В методе параллельно оптимизируется и топология ИНС, и ее параметры: пороги, весовые коэффициенты. Метод основан на поведенческо-ориентированном подходе к проектированию нейронных систем. Изначально он был разработан для изучения сложной динамики в сенсорно-двигательных системах автономных роботов и программных агентов. 
Главным достоинствам метода является его простота реализации и хорошая производительность для ИНС маленького и среднего размера. Стоит отметить, что в $\mathrm{ENS}^{3}$ не используется оператор кроссинговера, что можно отнести к недостатком, так как чаще всего, при хорошем подборе оператора скрещивания, можно добиться существенного сокращения времени эволюции и ускорить формирование оптимальной особи. Как ранее говорилось, метод ENS ${ }^{3}$ также не допускает различного вида функций активации нейронов, что также можно отнести к недостаткам. Кроме того, в методе фиксированы вероятности мутации для нейронов и связей, что сокращает пространство поиска: возможные эффективные конфигурации ИНС не будут сформированы в связи с ограниченной низкой вероятностью мутации параметров нейрона в текущей популяции.

DXNN (Deus Ex Neural Network, оптимизация нейронной сети) метод для раздельной модификации топологии и весовых коэффициентов ИНС, основанный на культурной эволюции, а именно меметичности. Метод был предложен Джином Шер в 2011 г [11].

В зависимости от реализации метод DXNN поддерживает прямой и косвенный способ кодирования хромосом. Для эволюции топологии в методе предусмотрен этап глобального поиска, в то время как на этапе локального поиска оптимизируются только весовые коэффицииенты.

Последовательная модификация топологии и весов позволяет определить по какой причине особь показывает низкое значение приспособленности: из-за неудачно сформированной топологии или неправильно подобранных весовых коэфффициентов. Стоит отметить, что в большинстве нейроэволюционных методов операторы, отвечающие за изменение весовых коэффициентов, применяются случайным образом ко всем нейронам ИНС, хотя вероятность оптимизации новых или требующих донастройки нейронов достаточно низкая.

Данный метод, ввиду использования меметичности, позволяет оптимизировать весовые коэффицциенты только недавно измененных или добавленных нейронов, при этом никак не изменяя топологию, полученную на предыдущих итерациях. Но есть и недостатки метода DXNN: отсутствие операторов мутации отвечающих за удаление связей и нейронов приводит к усложнению топологии ИНС и увеличению количества нейронов; метод оптимизирует только весовые коэффициенты из всех возможных параметров ИНС. При решении сложных задач данным методом на формирование оптимального решения затрачивается большое количество ресурсов и времени.

ICONE (Interactively Constrained Neuro-Evolution, динамическая нейроэволюция с ограничениями) - метод использует прямое кодирование с наложением ограничений для формирования ИНС необходимой топологии, не требует декодирования генотипа в фенотип. Метод разработан Кристианом Ремписом и Фрэнком Паземаном в 2012 г.

B метод ICONE геномы представляют собой полноценные ИНC, состоящие из простых элементов: нейронов, связей, групп нейронов и нейромодулей. Каждый из таких элементов имеет ряд параметров, предназначенных для оптимизации: пороговое значение, функция активации, весовой коэффицциент. Помимо настраиваемых параметров каждому элементу соотнесен список свойств, предназначенных для настройки процесса эволюции для данной особи (хранится в виде ключ-значение). Метод предполагает использование мутации добавления и удаления нейронов и связей, исключение составляют специальные нейроны с метками «вход», «выход».

Процесс эволюции, реализованный в методе ICON, требует вмешательства эксперта для установления ограничений на связи и другие значения параметров ИНС. Это свойство можно отнести как к достоинствам, так и к недостаткам, так как поиск решения (успех эволюции) во многом будет зависеть от опыта эксперта.

\section{Заключение}

Нейроэволюционные методы можно сравнить по следующим характеристикам: по наличию каких-либо ограничений, по виду используемых методов эволюции, по изменению параметров и топологии ИНС, по набору используемых генетических операторов.

В таблице 1 представлено сравнение методов по ограничениям, которые накладываются на параметры и топологию ИНС:

- на параметры ИНС (вид функции активации, пороговые значения, применение фиксированных значений);

- на топологию ИНС (количество возможных слоев, наличие обратных связей, фиксированная топология);

- отсутствие ограничений.
В таблице 2 представлено сравнение ключевых характеристик методов, а также кратко указаны основные особенности.

Таблица 1 - Сравнение методов по наличию ограничений

\begin{tabular}{|c|c|c|}
\hline \multirow{2}{*}{ Метод } & \multicolumn{2}{|c|}{ Вид ограничения } \\
\hline & Параметры & Топология \\
\hline ENS3 & Вид функции активации & Нет \\
\hline NEAT & Нет & $\begin{array}{c}\text { Исходная популяция: только } \\
\text { полносвязные ИНС; количество } \\
\text { нейронов фиксировано }\end{array}$ \\
\hline SANE & Вид функции активации & $\begin{array}{c}\text { ИНС прямого распространения } \\
\text { с одним скрытым слоем }\end{array}$ \\
\hline ESP & Вид функции активации & Полносвязные ИНС \\
\hline CNE & $\begin{array}{l}\text { Вид функции активации } \\
\text { и пороги фиксированы }\end{array}$ & $\begin{array}{c}\text { Топология фиксирована } \\
\text { и задается вначале }\end{array}$ \\
\hline EPNet & Вид функции активации & ИНС прямого распространения \\
\hline NES & Вид функции активации & $\begin{array}{c}\text { Топология фиксирована } \\
\text { и задается вначале }\end{array}$ \\
\hline EANT & Нет & Нет \\
\hline ICONE & Нет & Нет \\
\hline
\end{tabular}

Научный интерес вызывают методы, у которых отсутствуют какие-либо ограничения, что расширяет область их использования для ИНС любой топологии вне зависимости от вида и параметров, что, в свою очередь, позволяет наиболее полно исследовать пространство поиска и рассмотреть нейросети всех возможных конфигураций для формирования оптимального решения поставленной задачи, если это решение не требует минимального времени.

Таблица 2 - Сравнительные характеристики нейроэволюционных методов

\begin{tabular}{|c|c|c|c|c|}
\hline \multirow{2}{*}{ Метод } & \multirow{2}{*}{\begin{tabular}{|c|} 
Метод \\
эво- \\
люции
\end{tabular}} & \multicolumn{2}{|c|}{$\begin{array}{l}\text { Модифицируемое } \\
\text { свойство }\end{array}$} & \multirow{2}{*}{ Особенности } \\
\hline & & Параметры & Топология & \\
\hline ENS $^{3}$ & ЭА & $\begin{array}{l}\text { Пороги, } \\
\text { веса }\end{array}$ & Да & $\begin{array}{l}\text { Коэволюция, стохастические } \\
\text { генетические операторы, } \\
\text { эволюция без кроссинговера }\end{array}$ \\
\hline NEAT & ГА & Beca & Да & $\begin{array}{c}\text { Исторические маркеры } \\
\text { (инновационные числа), } \\
\text { видовая специализация, } \\
\text { использование синапсисаи } \\
\text { гомологичных генов }\end{array}$ \\
\hline SANE & ГА & Beca & Да & $\begin{array}{l}\text { Кооперация, коэволюция, } \\
\text { специализация, популяции } \\
\text { нейронов и шаблонов ИНС }\end{array}$ \\
\hline ESP & ГА & Beca & Нет & $\begin{array}{l}\text { Кооперация, коэволюция, } \\
\text { специализация, популяции } \\
\text { нейронов и шаблонов ИНС }\end{array}$ \\
\hline CNE & $\ulcorner A$ & Beca & Нет & $\begin{array}{c}\text { Структура ИНС } \\
\text { задается заранее, } \\
\text { классическая реализация } \\
\text { нейроэволюции } \\
\end{array}$ \\
\hline EPNet & ГА & Beca & $\begin{array}{c}\text { Да, } \\
\text { ограничено }\end{array}$ & $\begin{array}{c}\text { Использование метода } \\
\text { обучения нейросетей МОРО }\end{array}$ \\
\hline NES & ГА & Beca & Нет & $\begin{array}{c}\text { Использование операторов } \\
\text { кроссинговера и мутации SB } \\
\text { MAC и TVM; настройка пара- } \\
\text { метров генетических операто- } \\
\text { ров в ходе эволюции }\end{array}$ \\
\hline EANT & $Э A+Г A$ & Beca & Да & $\begin{array}{c}\text { Гибридная схема } \\
\text { кодирования } \\
\text { и генетические операторы }\end{array}$ \\
\hline ICONE & ЭА & $\begin{array}{c}\text { Вид функ- } \\
\text { ции актива- } \\
\text { ции, пороги, } \\
\text { веса }\end{array}$ & Да & $\begin{array}{c}\text { Ограничивающие маркеры, } \\
\text { отсутствие преобразований } \\
\text { фенотип-генотип }\end{array}$ \\
\hline
\end{tabular}

В настоящее время все больше методы нейроэволюции применяются в областях, в которых главным учителем выступает внешняя среда, с которой приходится взаимодействовать и получать опыт от такого взаимодействия. В задачах, где четко определена архитектура 
ИНС или есть собственные ограничения, можно использовать методы модифицирующие только весовые и пороговые значения: CNE, ESP, NES. Самым простым является метод CNE, но эксперименты показывают, что данный метод хорошо справляется с задачей управления агентами в мультиагентной системе [12].

Метод NEAT все больше используется для обучения ИНС в задачах управления поведением с обратным откликом: система предупреждения столкновения в автомобилях, управление роботами [13].

\section{Список цитированных источников}

1. Бессонов, А. А. Эволюционирующие искусственные нейронные сети прямого распространения: архитектуры, обучение, применения [текст] : дис. ... док. тех. наук : 05.13.23 / Бессонов А. А. Харьков., 2017. - $436 \mathrm{c}$.

2. Черноруцкий, И. Г. Методы принятия решений: учебное пособие / И. Г. Черноруцкий. - СПб. : БХВ-Петербург, 2005. - 416 с.

3. Хлопкова, О.А. Методы и алгоритмы интеллектуализации принятия решений в условиях неопределенности на базе аппарата нейронных сетей и эволюционного моделирования [текст] : дис. ... канд. тех. наук : 05.13.17 / Хлопкова О. А. - М., 2016. - 157 с.

4. Belew R. Evolving Networks: Using the Genetic Algorithm with Connectionist Learning/ R. Belew, J. Mcinerney, N. N. Shraudolph // Computer Science \& Engr. Dept - Univ. California at San Diego, 1990. - P. 40.

5. Riessen, G. A. PEPNet: parallel evolutionary programming for constructing artificial neural networks / G. A. Riessen, G. J. Williams, X. Yao // Berlin: 6th International Conference on Evolutionary Programming, 1997. - P. 35-45.

6. Moriarty, D. Forming neural networks through efficient and adaptive coevolution / D. Moriarty, R. Miikkulainen // Evolutionary Computation, 1997. - No 5. - P. 373-399.

7. Stanley, K. O. Evolving neural networks through augmenting topologies / K. O. Stanley, R. Miikkulainen // Evolutionary Computation, 2002. - Vol 10. - No 2. - P. 99-127.

8. Khayer Azad, M. A. On Comparison between Evolutionary Programming Network based Learning and Novel Evolution Strategy Algorithm-based Learning / Md. Shafiqul Islam and M. M. A. Hashem // Computer Science \& Engineering Discipline. Bangladesh: Khulna University of Engineering and Technology - 2003. - P. 213-218.

9. Kassahun, Y, Common Genetic Encoding for Both Direct and Indirect Encodings of Networks. / M. Edgington, J. H. Metzen, G. Sommer, F. Kirchner // In Proceedings of the Genetic and Evolutionary Computation Conference (GECCO) - 2007. - P. 1029-1036.

10. Pasemann, F. Evolving Structure and Function of Neurocontrollers / F. Pasemann, U. Steinmetz, U. Dieckmann // Washington: CEC 99: Proceedings of the 1999 Congress on Evolutionary Computation, 1999. - P. 22-29.

11. Sher, G. I. Evolving Chart Pattern Sensitive Neural Network Based Forex Trading Agents / G. I. Sher // Neural and Evolutionary Computing, Computational Engineering, Finance, and Science. - 2011. P. 47-68.

12. Давидюк, Ю. И. Эволюционный метод управления агентами В многоагентной системе / Ю. И. Давидюк // Вестник Брестского государственного технического университета. - Брест: БрГТУ 2020. - № 5: Физика, математика, информатика.- С. 2-4.

13. Stanley, K. O. Real-time neuroevolution in the NERO videogame/ K. O. Stanley, B. D. Bryant, R. Miikkulainen // Evolutionary Computation, 2005. - Vol 9. - No 6. - P. 653-668.

\section{References}

1. Bessonov, A. A. Evolyucioniruyushchie iskusstvennye nejronnye seti pryamogo ras pprostraneniya: arhitektury, obuchenie, primeneniya [tekst] : dis. ... dok. tekh. nauk : 05.13.23 / Bessonov A. A. - Har'kov. 2017. $-436 \mathrm{~s}$.

2. CHernoruckij, I. G. Metody prinyatiya reshenij: uchebnoe posobie / I. G. CHernoruckij. - SPb. : BHV-Peterburg, 2005. - $416 \mathrm{~s}$.

3. Hlopkova, O. A. Metody i algoritmy intellektualizacii prinyatiya reshenij $v$ usloviyah neopredelennosti na baze apparata nejronnyh setej i evolyucionnogo modeli-rovaniya [tekst] : dis. ... kand. tekh. nauk : 05.13.17 / Hlopkova O. A. - M., 2016. - 157 s.

4. Belew R. Evolving Networks: Using the Genetic Algorithm with Connectionist Learning/ R. Belew, J. Mcinerney, N. N. Shraudolph// Computer Science \& Engr. Dept - Univ. California at San Diego, 1990. - P. 40.

5. Riessen, G. A. PEPNet: parallel evolutionary programming for constructing artificial neural networks / G. A. Riessen, G. J. Williams, $X$. Yao // Berlin: 6th International Conference on Evolutionary Programming, 1997. - P. 35-45.

6. Moriarty, D. Forming neural networks through efficient and adaptive coevolution / D. Moriarty, R. Miikkulainen // Evolutionary Computation, 1997. - No 5. - P. 373-399.

7. Stanley, K. O. Evolving neural networks through augmenting topologies / K. O. Stanley, R. Miikkulainen // Evolutionary Computation, 2002. - Vol 10. - No 2. - P. 99-127.

8. Khayer Azad, M. A. On Comparison between Evolutionary Programming Network based Learning and Novel Evolution Strategy Algorithm-based Learning / Md. Shafiqul Islam and M. M. A. Hashem // Computer Science \& Engineering Discipline. Bangladesh: Khulna University of Engineering and Technology - 2003. - P. 213-218.

9. Kassahun, $Y$, Common Genetic Encoding for Both Direct and Indirect Encodings of Networks. / M. Edgington, J. H. Metzen, G. Sommer, F. Kirchner // In Proceedings of the Genetic and Evolutionary Computation Conference (GECCO) - 2007. - P. 1029-1036.

10. Pasemann, F. Evolving Structure and Function of Neurocon-trollers / F. Pasemann, U. Steinmetz, U. Dieckmann // Washington: CEC 99: Proceedings of the 1999 Congress on Evolutionary Computation, 1999. - P. 22-29.

11. Sher, G. I. Evolving Chart Pattern Sensitive Neural Network Based Forex Trading Agents / G. I. Sher // Neural and Evolutionary Computing, Computational Engineering, Finance, and Science. - 2011. P. 47-68.

12. Davidyuk, YU. I. Evolyucionnyj metod upravleniya agentami v mnogoagentnoj siาsteme / YU. I. Davidyuk // Vestnik Brestskogo gosudarstvennogo tekhnicheskogo universite 7 ta. - Brest: BrGTU - 2020. № 5: Fizika, matematika, informatika. - C. 2-4.

13. Stanley, K. O. Real-time neuroevolution in the NERO videogame/ K. O. Stanley, B. D. Bryant, R. Miikkulainen // Evolutionary Computation, 2005. - Vol 9. - No 6. - P. 653-668.

Материал поступил в редакцию 07.05.2021 